J. Clin. Chem. Clin. Biochem.

Vol. 18. 1980, pp. 901-908

\title{
Der Einfluß von Insulin auf den Stoffwechsel von isolierten Glomerula der Ratte ${ }^{1}$ )
}

\author{
Von J. Oldendörp und H. Reinauer
}

Lehrstuhl für klinische Biochemie und biochemische Abteilung des Diabetes-Forschungsinstituts an der Universität Dïsseldorf

(Eingegangen am 7. Dezember 1979/14. Mai 1980)

Zusammenfassung: Isolierte Glomerula wurden aus den Nieren von stoffwechselgesunden und diabetischen Ratten gewonnen und ihre Morphologie und ihre metabolische Leistungsfähigkeit untersucht. Der DNA- und Proteingehalt der isolierten Glomerula war bei chronisch diabetischen Tieren signifikant erhöht und die DNA/Protein-Relation verändert. Diese Änderungen konnten durch Insulinbehandlung der diabetischen Ratten in vivo verhindert werden. Insulin hatte keinen Einfluß auf den Abbau von Glucose in den Glomerula von normalen und diabetischen Ratten. Die Proteinsynthese ist in den Glomerula von akut diabetischen Ratten unverändert, steigt jedoch im chronischen Diabetes signifikant an. Die RNA-Synthese hingegen ist im akuten Diabetes auf ein Drittel vermindert, steigt aber bei chronischem Diabetes ebenfalls signifikant an. Während in den Glomerula von Normaltieren sowohl die Proteinsynthese als auch die RNA-Synthese durch Insulinzugabe in vitro gesteigert werden kann, fehlt dieser Effekt in den Glomerula von diabetischen Ratten. Eine Insulinresistenz in vitro ist somit festzustellen. Nur die Insulinsubstitution in vivo verhindert die Änderungen der Protein- und RNA-Synthese. Der Einbau von Lysin und Uridin war in den Glomerula von Normaltieren zusätzlich abhängig von der Glucosekonzentration.

Aus den Untersuchungen wird gefolgert, daß unter Insulinmangel und erhöhter Blutzuckerkonzentration charakteristische Veränderungen des Protein- und RNA-Stoffwechsels auftreten, die auf die morphologisch und biochemisch faßbaren Strukturveränderungen bezogen werden können.

\section{The effect of insulin on the metabolism of isolated rat glomeruli}

Summary: Glomeruli isolated from the kidneys of metabolically normal and diabetic rats were investigated morphologically and metabolically. Glomeruli from chronically diabetic animals showed significantly increased contents of DNA and protein, and an altered DNA/protein ratio. These changes were prevented by insulin treatment in vivo of diabetic rats. Insulin had no effect on glucose degradation by the glomeruli of normal or diabetic rats. Protein synthesis is unchanged in the glomeruli from acutely diabetic rats, but shows a significant increase in those from chronically diabetic animals. In the glomeruli of normal animals, both protein synthesis and RNA synthesis are increased in vitro by insulin, but this effect is absent from the glomeruli of diabetic rats. Under in vitro conditions there is a resistance to insulin. Changes in protein and RNA synthesis can be prevented by insulin only when it is administered in vivo. In addition, the incorporation of lysine and uridine in the glomeruli of normal animals was dependent on the glucose concentration.

It is concluded from these results that insulin deficiency and increased blood sugar level are accompanied by characteristic changes in the metabolism of protein and RNA, which are related to morphologically and biochemically detectable structural changes.

\section{Einfựhnung}

Die diabetische Glomerulosklerose ist morphologisch charakterisiert durch eine diffuse Verdickung der Basalmembran, durch Vergrößerung der Glomerula und Ver-

\footnotetext{
1) Die Untersuchungen wurden mit Unterstützung der Paul-KuthStiftung durchgeführt.
}

mehrung des Mesangiums mit terminaler Hyalinisierung. Die Basalmembranverdickung wurde nicht nur beim Menschen, sondern auch bei Versuchstieren mit chemisch induziertem (1-2) oder genetischem Diabetes (3) nachgewiesen.

Uber die metabolischen Ursachen der genannten Basalmembranveränderungen in den Glomerula gibt es nur 
Hypothesen. Die Frage, ob die Synthese der Basalmembranproteine quantitativ verändert und/oder ihr Abbau vermindert sind, wurde noch nicht schlüssig geklärt.

Bisher wurden vorwiegend-Befunde an einzelnen Enzymen, an der Proteinsynthese und anderen Stoffwechselgrößen erhoben und zur Erklärung der diabetischen Glomerulosklerose herangezogen.

So wurde eine erhöhte Aktivität der Lysin-Hydroxylase (EC 1.14.11.4) und der UDP-Glucose-Transferase im Diabetes gefunden (4-9).

Eine Erhöhung der Proteinsynthese in Mikrosomen- bzw. Ribosomenpräparationen aus der Nierenrinde von diabetischen Versuchstieren und eine deutlich erhöhte Kollagensynthese wurden gemessen (10-11). Der erhöhte Lysin-Einbau, die erhöhte Hydroxylysin-Synthese und das zugunsten des Hydroxylysin verschobene "Lysin/Hydroxylysin-Verhältnis in isolierten Glomerula und die Normalisierung durch die Insulintherapie ergänzen diese $\mathrm{Be}$ funde (12). Dementsprechend war die Aktivität der Prolin-Hydroxylase (EC 1.14.11.2) und der Lysin-Hydroxylase in der Nierenrinde von Streptozotocin-diabetischen Ratten erhöht (8).

Widersprüchliche Befunde liegen über die quantitative Aminosäurezusammensetzung der glomerulären Basalmembran vor (13-17).

Auch der Einfluß von Insulin in vitro auf die Oxidation und den Einbau von Glucose in isolierten Glomerula von Alloxan-diabetischen Tieren wurde untersucht. Insulin hatte keinen Effekt, jedoch wurden sowohl die Oxidation als auch der Einbau von Glucose in Glomerula von normalen und diabetischen Tieren durch Erhöhung der Glucosekonzentration im Inkubationsmedium gesteigert (18-19). Ein Teil der sich widersprechenden Ergebnisse wurde an der ganzen Niere oder der Nierenrinde erhoben. Bei diesen Untersuchungen überwiegt aber das tubuläre Zellsystem, so daß diese Ergebnisse nur eine geringe Aussagekraft für glomeruläre Stoffwechselveränderungen besitzen. Einigkeit besteht auch darüber, daß Enzymaktivitätsmessungen im Gewebshomogenat unter optimierten Bedingungen die aktuellen Gegebenheiten im Stoffwechsel und den tatsächlichen Substratumsatz nicht wiedergeben können.

Aus den genannten Gründen wurde der Stoffwechsel des Zellsystems der isolierten Glomerula von normalen und diabetischen Ratten verglichen. Im Rahmen dieser Untersuchungen wurden der Kohlenhydrat-, Protein- und Ribonucleinsäurestoffwechsel unter Berücksichtigung der Insulinsensitivität untersucht.

\section{Material und Methoden}

Männliche Wistar-Ratten (250-300 g) wurden nach 20 Stunden Hunger mit Äther narkotisiert, die Nieren wurden entfernt, dekapsuliert und halbiert. Die Isolation der Glomerula erfolgte in Anlehnung an die Siebmethode von Krakower \& Greenspon (20). Die Nierenhälften wurden duch ein Stahlsieb mit $280 \mu \mathrm{m} \mathrm{Ma-}$ schenweite gerieben. Anschließend wurde mit eiskaltem Medium TC 199 (Serva Feinbiochemica, Heidelberg: TC Medium 199 mit Earle's Salzen ohne Glucose, $L$-Lysin, ATP, Natriumacetat und Tween 80) nachgespült. Zur Abtrennung grober Gewebsbestandteile wurde der Gewebebrei durch ein Kunststoffnetz von $180 \mu \mathrm{m}$ Maschenweite gegossen und die Glomerula dann auf einem weiteren Kunststoffnetz von $100 \mu \mathrm{m}$ Maschenweite aufgefangen und in eiskaltem Medium TC 199 suspendiert. Zur höheren Konzentrierung der Glomerulumsuspension folgte eine Sedimentation von 30 Minuten im Eisbad mit anschließendem Absaugen des Überstandes. Danach wurden die Glomerula sofort zur Inkubation verwendet. Die Ausbeute betrug etwa 10000 Glomerula pro Niere. Die Verunreinigung durch Tubulusfragmente lag bei duchschnittlich $2 \%$. Etwa $20 \%$ der Glomerula besaßen noch eine intakte Bowman' Kapsel. Die Vitalitätsprüfung erfolgte durch Anfärbung mit Trypanblau, durch Licht- und Elektronenmikroskopie (Abb. 1) und aufgrund metabolischer Parameter.

\section{Erzeugung des Diabetes (Tab. 1)}

Der akute Diabetes wurde durch eine Streptozotocin-Injektion von $80 \mathrm{mg} / \mathrm{kg}$ Körpergewicht intravenös erzeugt und der Glomerulumstoffwechsel nach einer Diabetesdauer von 3 Tagen untersucht. Der Blutzucker lag über $350 \mathrm{mg} / \mathrm{dl}$. Es bestand eine massive Glucosurie mit Ketonurie.

Für den chronischen Diabetes erhielten die Tiere eine intraperitoneale Streptozotocin-Injektion von $80 \mathrm{mg} / \mathrm{kg}$ Körpergewicht und wurden nach einer Diabetesdauer von 4 Wochen untersucht.

Eine Gruppe dieser chronisch diabetischen Tiere erhielt täglich Insulin (10 U/kg Körpergewicht) über die gesamte Versuchsdauer (chronischer Diabetes mit Dauertherapie). Der Blutzuckerspiegel betrug nüchtern $100 \mathrm{mg} / \mathrm{dl}$. Es bestand eine leichte Glucosurie ohne Ketonurie.

Eine weitere Gruppe (chronischer Diabetes mit Teiltherapie) erhielt das Insulin erst nach einem therapiefreien Intervall von 14 Tagen. Dabei lagen die Blutzuckernüchternwerte am Ende der Versuchsdauer bei $110 \mathrm{mg} / \mathrm{dl}$

In der dritten Gruppe wurde kein Insulin appliziert. Diese Gruppe erreichte duchschnittliche Blutzuckerwerte von $480 \mathrm{mg} / \mathrm{dl}$ und eine massive Glucosurie und Ketonurie (chronischer Diabetes).

Zur Bestimmung der Oxidationsrate wurde das produzierte ${ }^{14} \mathrm{CO}_{2}$ durch Hyamine ${ }^{\circledR}$ absorbiert und im Flüssigkeitsszintillationszähler gezählt.

\section{Oxidation von Glucose:}

Endkonzentration von Glucose: $10 \mathrm{mmol} / \mathrm{l}$ Spezifische Radioaktivität von [ $\mathrm{U}^{-14} \mathrm{C}$ ] Glucose: $37 \mathrm{GBq} / \mathrm{mol}$ Oxidation und Einbau von Glucuronat:

Endkonzentration von Glucuronat: $5 \mathrm{mmol} / 1$

Spezifische Radioaktivität von [U- ${ }^{14}$ C] Glucuronat: $37 \mathrm{GBq} / \mathrm{mol}$

Oxidation und Einbau von Xylit:

Endkonzentration von Xylit: $5 \mathrm{mmol} / \mathrm{l}$

Spezifische Radioaktivität von [U-14 C]Xylit: $37 \mathrm{GBq} / \mathrm{mol}$

- Bei der Bestimmung der Einbaurate wurde das Sediment nach Säurefällung dreimal gewaschen, in Hyamine ${ }^{\circledR}$ im Flüssigkeitsszintillationszähler bestimmt.

Einbau von Lysin:

Endkonzentration von Glucose: $10 \mathrm{mmol} / \mathrm{l}$ oder wie angegeben Endkonzentration von Lysin: $500 \mu \mathrm{mol} / 1$ Spezifische Radioaktivität von [U-14 C]Lysin: $370 \mathrm{GBq} / \mathrm{mol}$

Einbau von Uridin:

Endkonzentration von Glucose: $10 \mathrm{mmol} / \mathrm{l}$ oder wie angegeben Endkonzentration von Uridin: $4 \mu \mathrm{mol} / 1$

Spezifische Radioaktivität von [U-14 C]Uridin: $18,5 \mathrm{TBq} / \mathrm{mol}$

Die Inkubation der Glomerula erfolgte jeweils in Dreifachansätzen bei $37^{\circ} \mathrm{C}$ im Schüttelwasserbad über 60 und 120 Minuten. Das Endvolumen der Ansätze betrug 0,5 ml, die Glomerulumsuspension (2500-7000 Glomerula) wurde in einem Volumen von $0,2 \mathrm{ml}$ zuğegeben. 


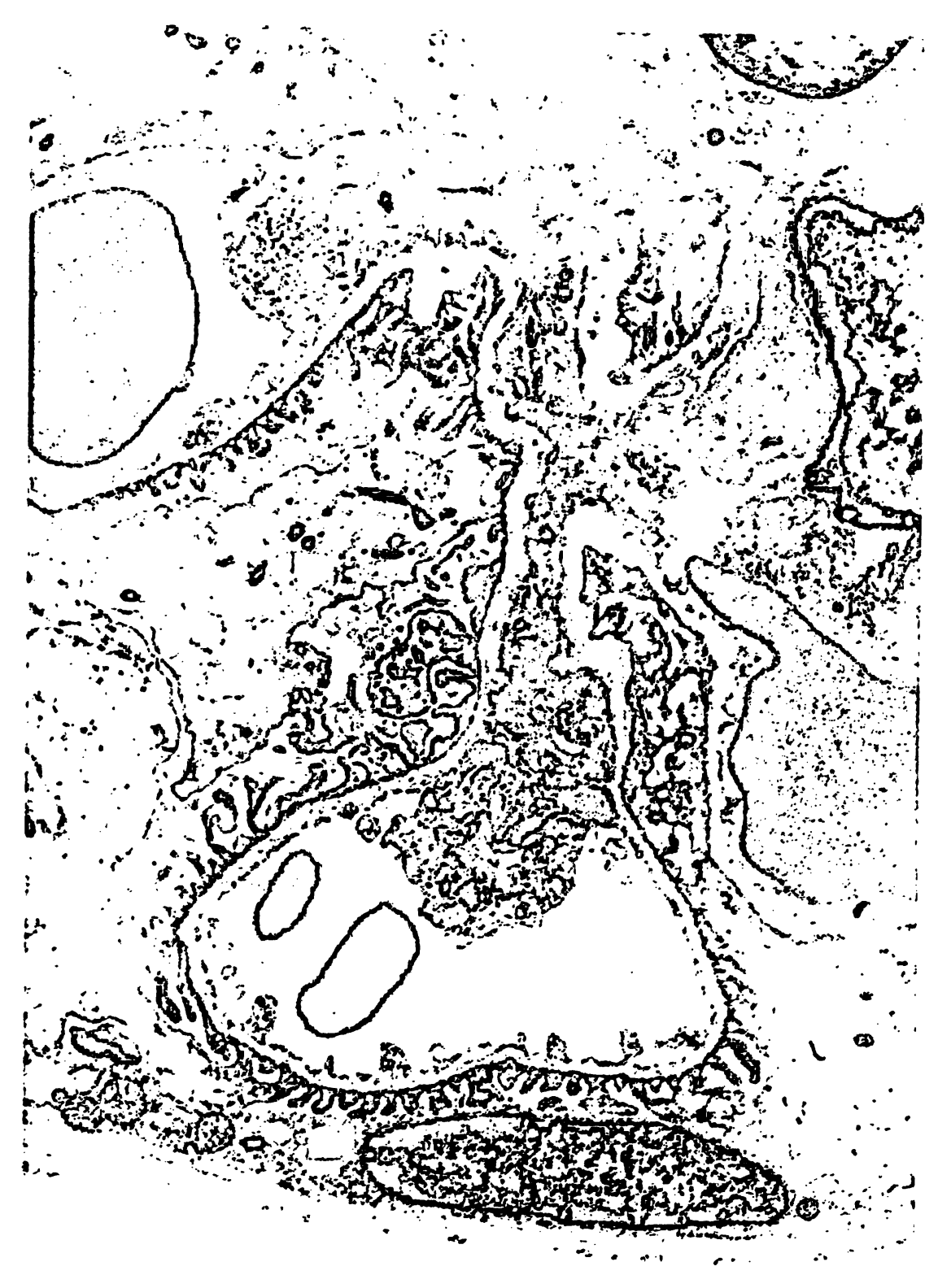

Abb. 1. Ultrastruktur der isolierten Glomerula.

Die elektronenmikroskopische Untersuchung zeigt den morphologischen Erhaltungszustand des Zellsystems. Vergrößerung 6000:1.

Die Insulinsensitivität wurde durch eine Insulinendkonzentration von $50 \mathrm{mU} /$ Ansatz (100 U/1) geprüft, nachdem Vorversuche einen hohen Insulinabbau ergeben hatten.

Der Proteingehalt der Glomerula wurde nach Lowry et al. (21) bestimmt. Die Bestimmung des DNA-Gehaltes erfolgte nach Burton (22).

Die statistischen Berechnungen wurden mit dem t-Test nach Student ausgeführt.

\section{Ergebnisse}

DNA- und Proteingehalt der isolierten Glomerula waren im akuten Diabetes gegenüber den Normaltieren nicht verändert (Tab. 2). Bei den chronisch diabetischen Tieren war eine signifikante Steigerung $(2 p<0,001)$ des DNA- und Proteingehaltes auf das Doppelte bzw. Zweieinhalbfache festzustellen, woraus eine Verschiebung des DNA/Protein-Verhältnisses von 1:20 auf 1:25 resultierte.
Insulinsubstitution in vivo führt zur signifikanten Verringerung $(2 p<0,001)$ des DNA- und Proteingehaltes, ohne jedoch eine vollständige Normalisierung $z u$ bewirken. Es fanden sich keine Unterscniede zwischen Dauerund Teil therapie.

Die Stoffwechselleistung der isolierten Glomerula, dargestellt an Glucose-Oxidation und Lysin-Einbau, war bei normalen und diabetischen Tieren linear in Abhängigkeit von der Zeit und der eingesetzten Gewebemenge. Die Abbildung 2 zeigt dies für die Glucose-Oxidation in Glomerula von Normaltieren.

Beim Abbau von Glucose zu $\mathrm{CO}_{2}$ waren keine Unterschiede in den isolierten Glomerula von normalen und akut diabetischen Tieren nachweisbar. Insulin in vitro 
Tab. 1. Körpergewicht, Blutzuckerkonzentration, Harnzucker und Ketonurie bei chronisch diabetischen Ratten. Harnzucker und Ketonurie wurden mit Gluketur ${ }^{\circledR}$-Test bestimmt.

\begin{tabular}{|c|c|c|c|c|c|c|c|c|c|c|c|c|c|c|c|c|}
\hline & \multicolumn{2}{|c|}{ 1. Woche } & \multirow{3}{*}{$\begin{array}{l}\text { Harn- } \\
\text { zuk- } \\
\text { ker }\end{array}$} & \multirow{3}{*}{$\begin{array}{l}\text { Ke- } \\
\text { ton- } \\
\text { urie }\end{array}$} & \multicolumn{3}{|c|}{ 2. Woche } & \multirow{3}{*}{$\begin{array}{l}\mathrm{Ke}- \\
\text { ton- } \\
\text { urie }\end{array}$} & \multicolumn{2}{|c|}{ 3. Woche } & \multirow{3}{*}{$\begin{array}{l}\text { Harn- } \\
\text { zuk- } \\
\text { ker }\end{array}$} & \multirow{3}{*}{$\begin{array}{l}\mathrm{Ke}- \\
\text { ton- } \\
\text { urie }\end{array}$} & \multicolumn{2}{|c|}{ 4. Woche } & \multirow{3}{*}{$\begin{array}{l}\text { Harn- } \\
\text { zuk- } \\
\text { ker }\end{array}$} & \multirow{3}{*}{$\begin{array}{l}\text { Ke- } \\
\text { ton- } \\
\text { urie }\end{array}$} \\
\hline & $\begin{array}{l}\text { Kör- } \\
\text { per- } \\
\text { ge- } \\
\text { wicht }\end{array}$ & $\begin{array}{l}\text { Blut- } \\
\text { zuk- } \\
\text { ker }\end{array}$ & & & $\begin{array}{l}\text { Kör- } \\
\text { per } \\
\text { ge- } \\
\text { wicht }\end{array}$ & $\begin{array}{l}\text { Blut- } \\
\text { zuk- } \\
\text { ker }\end{array}$ & $\begin{array}{l}\text { Harn- } \\
\text { zuk- } \\
\text { ker }\end{array}$ & & $\begin{array}{l}\text { Kör- } \\
\text { per- } \\
\text { ge- } \\
\text { wicht }\end{array}$ & $\begin{array}{l}\text { Blut- } \\
\text { zuk- } \\
\text { ker }\end{array}$ & & & $\begin{array}{l}\text { Kör- } \\
\text { per- } \\
\text { ge- } \\
\text { wicht }\end{array}$ & $\begin{array}{l}\text { Blut } \\
\text { zuk- } \\
\text { ker }\end{array}$ & & \\
\hline & (g) & (mg/dl) & & & (g) & \multicolumn{2}{|c|}{ (mg/dl) } & & (g) & $(\mathrm{mg} / \mathrm{dl})$ & & & (g) & $(\mathrm{mg} / \mathrm{dl})$ & & \\
\hline $\begin{array}{l}\text { Chronischer Diabetes } \\
(n=15)\end{array}$ & $\begin{array}{l}253 \\
\pm 25\end{array}$ & $\begin{array}{l}400 \\
\pm 60\end{array}$ & +++ & + & $\begin{array}{l}220 \\
\pm 20\end{array}$ & $\begin{array}{l}450 \\
\pm 80\end{array}$ & +++ & ++ & $\begin{array}{l}200 \\
\pm 18\end{array}$ & $\begin{array}{l}460 \\
\pm 80\end{array}$ & +++ & +++ & $\begin{array}{l}190 \\
\pm 15\end{array}$ & $\begin{array}{l}480 \\
\pm 100\end{array}$ & +++ & +++ \\
\hline \multicolumn{17}{|l|}{$\begin{array}{l}\text { Körpergewicht } \\
=290 \pm 15 \mathrm{~g}\end{array}$} \\
\hline $\begin{array}{l}\text { Chronischer Diabetes } \\
\text { mit Teiltherapie } \\
(n=20)\end{array}$ & $\begin{array}{l}250 \\
\pm 20\end{array}$ & $\begin{array}{l}410 \\
\pm 50\end{array}$ & +++ & + & $\begin{array}{l}230 \\
\pm 22\end{array}$ & $\begin{array}{l}440 \\
\pm 80\end{array}$ & +++ & ++ & $\begin{array}{l}240 \\
\pm 25\end{array}$ & $\begin{array}{l}160 \\
\pm 60\end{array}$ & ++ & + & $\begin{array}{l}280 \\
\pm 22\end{array}$ & $\begin{array}{l}110 \\
\pm 30\end{array}$ & +++ & - \\
\hline $\begin{array}{l}\text { Körpergewicht } \\
=280 \pm 18 \mathrm{~g}\end{array}$ & & & & & & & & & & & & & & & & \\
\hline $\begin{array}{l}\text { Chronischer Diabetes } \\
\text { mit Dauertherapie } \\
(\mathrm{n}=20)\end{array}$ & $\begin{array}{l}280 \\
\pm 25\end{array}$ & $\begin{array}{l}180 \\
\pm 80\end{array}$ & + & - & $\begin{array}{l}300 \\
\pm 20\end{array}$ & $\begin{array}{l}100 \\
\pm 30\end{array}$ & + & - & $\begin{array}{l}310 \\
\pm 27\end{array}$ & $\begin{array}{l}100 \\
\pm 25\end{array}$ & + & - & $\begin{array}{l}315 \\
\pm 30\end{array}$ & $\begin{array}{l}100 \\
\pm 20\end{array}$ & + & - \\
\hline $\begin{array}{l}\text { Körpergewicht } \\
=280 \pm 16 \mathrm{~g}\end{array}$ & & & & & & & & & & & & & & & & \\
\hline
\end{tabular}

Tab. 2. DNA- und Proteingehalt von isolierten Glomerula.

\begin{tabular}{llll}
\hline & $\begin{array}{l}\text { DNA } \\
(\mu \mathrm{g} / 1000 \\
\text { Glomerula })\end{array}$ & $\begin{array}{l}\text { Protein } \\
(\mu \mathrm{g} / 1000 \\
\text { Glomerula) }\end{array}$ & $\begin{array}{l}\text { DNA: } \\
\text { Pro- } \\
\text { tein }\end{array}$ \\
\hline Kontrolle & $\begin{array}{l}8,16 \pm 0,56 \\
(\mathrm{n}=30)\end{array}$ & $\begin{array}{l}165 \pm 15 \\
(\mathrm{n}=30)\end{array}$ & $1: 20$ \\
Akuter Diabetes & $\begin{array}{l}8,16 \pm 0,87 \\
(\mathrm{n}=20)\end{array}$ & $\begin{array}{l}165 \pm 11 \\
(\mathrm{n}=20)\end{array}$ & $1: 20$ \\
Chronischer Diabetes & $\begin{array}{l}15,97 \pm 1,82 \\
(\mathrm{n}=10)\end{array}$ & $\begin{array}{l}401 \pm 25 \\
(\mathrm{n}=10)\end{array}$ & $1: 25$ \\
$\begin{array}{l}\text { Chronischer Diabetes } \\
\text { mit Teiltherapie }\end{array}$ & $\begin{array}{l}11,07 \pm 0,93 \\
(\mathrm{n}=10)\end{array}$ & $\begin{array}{l}234 \pm 32 \\
(\mathrm{n}=10)\end{array}$ & $1: 21$ \\
$\begin{array}{l}\text { Chronischer Diabetes } \\
\text { mit Dauertherapie }\end{array}$ & $\begin{array}{l}11,44 \pm 0,73 \\
(\mathrm{n}=10)\end{array}$ & $\begin{array}{l}234 \pm 18 \\
(\mathrm{n}=10)\end{array}$ & $1: 21$ \\
\hline
\end{tabular}

Tab. 3. Abbau zu $\mathrm{CO}_{2}$.

Insulinkonzentration: $100 \mathrm{U} / 1$

\begin{tabular}{llll}
\hline & $\begin{array}{l}\text { Glucose } \\
(\mathrm{nmol} / 1000 \\
\text { Glomerula } \times \mathrm{h})\end{array}$ & $\begin{array}{l}\text { Glucuronat } \\
(\mathrm{nmol} / 1000 \\
\text { Glomerula } \times \mathrm{h})\end{array}$ & $\begin{array}{l}\text { Xylit } \\
(\mathrm{nmol} / 1000 \\
\text { Glomerula } \times \mathrm{h})\end{array}$ \\
\hline Kontrolle & $\begin{array}{l}0,71 \pm 0,11 \\
(\mathrm{n}=10)\end{array}$ & $\begin{array}{l}0,27 \pm 0,07 \\
(\mathrm{n}=3)\end{array}$ & $\begin{array}{l}0,11 \pm 0,01 \\
(\dot{\mathrm{n}}=3)\end{array}$ \\
$\begin{array}{l}\text { Kontrolle } \\
+ \text { Insulin }\end{array}$ & $\begin{array}{l}0,82 \pm 0,11 \\
(\mathrm{n}=8)\end{array}$ & $\begin{array}{l}0,27 \pm 0,05 \\
(\mathrm{n}=3)\end{array}$ & $\begin{array}{l}0,12 \pm 0,02 \\
(\mathrm{n}=3)\end{array}$ \\
$\begin{array}{l}\text { Akuter } \\
\text { Diabetes }\end{array}$ & $\begin{array}{l}0,65 \pm 0,07 \\
(\mathrm{n}=5)\end{array}$ & $\begin{array}{l}0,26 \pm 0,07 \\
(\mathrm{n}=3)\end{array}$ & $\begin{array}{l}0,09 \pm 0,01 \\
(\mathrm{n}=3)\end{array}$ \\
$\begin{array}{l}\text { Akuter } \\
\text { Diabetes } \\
+ \text { Insulin }\end{array}$ & $\begin{array}{l}0,72 \pm 0,03 \\
(\mathrm{n}=5)\end{array}$ & $\begin{array}{l}0,25 \pm 0,05 \\
(\mathrm{n}=3)\end{array}$ & $\begin{array}{l}0,08 \pm 0,02 \\
(\mathrm{n}=3)\end{array}$ \\
\hline
\end{tabular}

hatte keinen Einfluß auf die Abbaurate von Glucose (Tab. 3).

Glucuronat und Xylit werden durçh das glomeruläre Żellsystem zu $\mathrm{CO}_{2}$ abgebaut (Tab. 3). Dieser Abbau ist durch
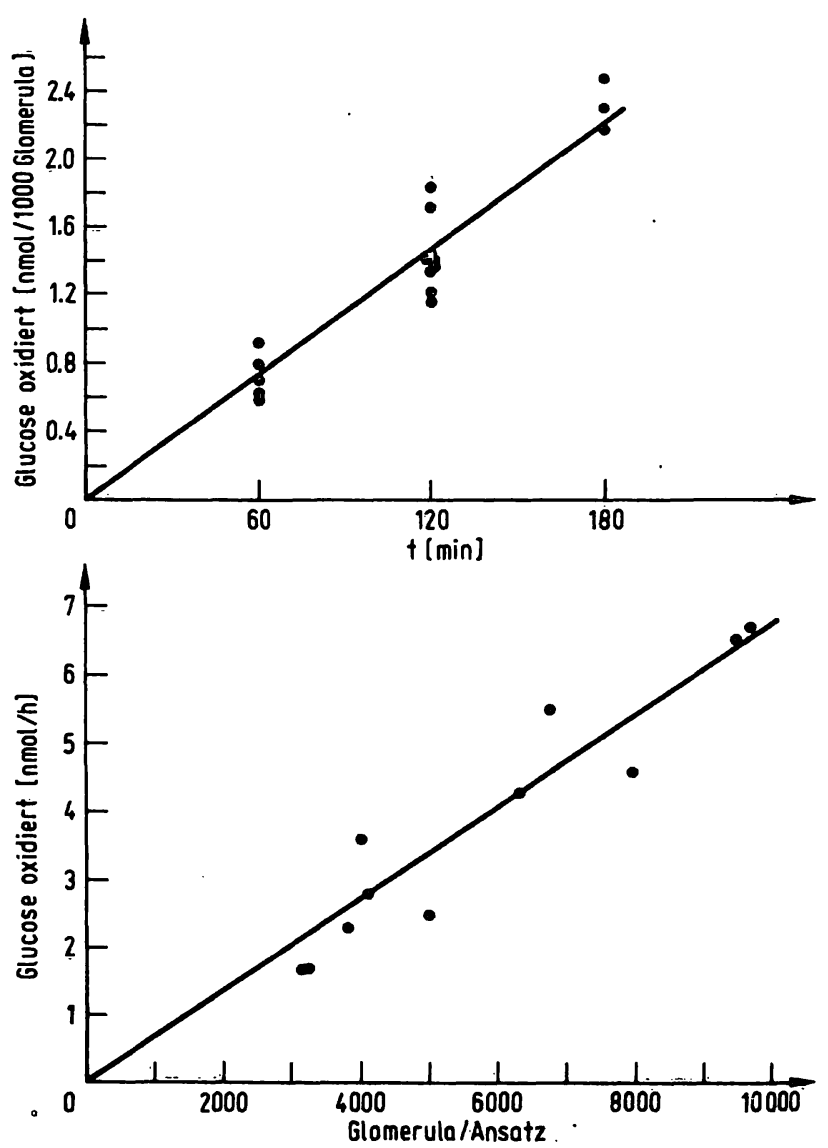

Abb. 2. Oxidation von Glucose in Abhängigkeit von der Zeit und der eingesetzten Gewebemenge durch das glomeruläre Zellsystem. 
Insulin in vitro und akuten Insulinmangel in vivo nicht zu beeinflußen. Auffallend ist, daß der Abbau von Xylit nur etwa die Hälfte des Glucuronat-Abbaues beträgt.

Glucuronat wird im Gegensatz zu Xylit auch in das säurefällbare Protein eingebaut (Tab. 4). Auch dieser Einbau zeigt keine Veränderung im akuten Diabetes und läßt sich auch durch Insulin in vitro nicht beeinflußen.

Der Einbau von Lysin in das säurefällbare Protein ist in den Glomerula von stoffwechselgesunden Tieren durch Insulin in vitro signifikant $(2 p<0,001)$ zu steigern (Tab. 4). In den Glomerula von akut diabetischen Tieren ist dieser Effekt nicht nachweisbar: Gegenüber den Normaltieren ist der Einbau von Lysin im akuten Diabetes unverändert. Beim chronischen Diabetes ist der LysinEinbau bezogen auf die Zahl der Glomerula signifikant $(2 p<0,001)$ erhöht. Insulinbehandlung nach therapiefreiem Intervall führt zu einer weiteren Steigerung, während sich der Lysin-Einbau bei durchgehender Insulinsubstitution wieder der Kontrolle annähert (Tab. 4).

Tab. 4. Einbau von Glucuronat, Lysin und Uridin in das säurefällbare Protein.

\begin{tabular}{|c|c|c|c|}
\hline & $\begin{array}{l}\text { Glucuronat } \\
\text { (nmol/1000 } \\
\text { Glomerula } \\
\times \text { h) }\end{array}$ & $\begin{array}{l}\text { Lysin } \\
\text { (nmol/1000 } \\
\text { Glomerula } \\
\times h \text { ) }\end{array}$ & $\begin{array}{l}\text { Uridin } \\
(\mathrm{Bq} / 1000 \\
\text { Glomerula } \\
\times \mathrm{h})\end{array}$ \\
\hline Kontrolle & $\begin{array}{l}0,10 \pm 0,02 \\
(n=3)\end{array}$ & $\begin{array}{l}0,086 \pm 0,005 \\
(n=8)\end{array}$ & $\begin{array}{l}6,30 \pm 0,13 \\
(n=5)\end{array}$ \\
\hline $\begin{array}{l}\text { Kontrolle } \\
+ \text { Insulin }\end{array}$ & $\begin{array}{l}\left.0,11 \pm 0,02^{1}\right) \\
(n=3)\end{array}$ & $\begin{array}{l}\left.0,125 \pm 0,021^{3}\right) \\
(n=6)\end{array}$ & $\begin{array}{l}\left.7,28 \pm 0,47^{4}\right) \\
(n=5)\end{array}$ \\
\hline $\begin{array}{l}\text { Akuter } \\
\text { Diabetes }\end{array}$ & $\begin{array}{l}\left.0,10 \pm 0,01^{1}\right) \\
(n=3)\end{array}$ & $\begin{array}{l}\left.0,086 \pm 0,006^{1}\right) \\
(n=5)\end{array}$ & $\begin{array}{l}\left.2,17 \pm 0,25^{3}\right) \\
(n=5)\end{array}$ \\
\hline $\begin{array}{l}\text { Akuter } \\
\text { Diabetes } \\
+ \text { Insulin }\end{array}$ & $\begin{array}{l}\left.0,10 \pm 0,03^{2}\right) \\
(n=3)\end{array}$ & $\begin{array}{l}\left.0,095 \pm 0,009^{2}\right) \\
(n=5)\end{array}$ & $\begin{array}{l}\left.2,13 \pm 0,33^{2}\right) \\
(n=5)\end{array}$ \\
\hline $\begin{array}{l}\text { Chronischer } \\
\text { Diabetes }\end{array}$ & n. b. & $\begin{array}{l}\left.0,105 \pm 0,007^{5}\right) \\
(n=5)\end{array}$ & $\begin{array}{l}\left.8,37 \pm 0,45^{6}\right) \\
(n=5)\end{array}$ \\
\hline $\begin{array}{l}\text { Chronischer } \\
\text { Diabetes mit } \\
\text { Teiltherapie }\end{array}$ & n. b. & $\begin{array}{l}\left.0,147 \pm 0,022^{7}\right) \\
(n=5)\end{array}$ & $\begin{array}{l}\left.6,77 \pm 0,95^{8}\right) \\
(n=5)\end{array}$ \\
\hline $\begin{array}{l}\text { Chronischer } \\
\text { Diabetes mit } \\
\text { Dauer- } \\
\text { therapie }\end{array}$ & n. b. & $\begin{array}{l}\left.0,104 \pm 0,018^{9}\right) \\
(n=5)\end{array}$ & $\begin{array}{l}6,12 \pm 0,07^{10} \\
(n=5)\end{array}$ \\
\hline
\end{tabular}

1) Nicht signifikant gegen Kontrolle.

2) Nicht signifikant gegen akuten Diabetes.

3) $2 \mathrm{p}<0 ; 001$ gègen Kontrolle.

4) $2 p<0,005$ gegen Kontrolle.

5) $2 \mathrm{p}<0,001$ gegen Kontrolle; $2 \mathrm{p}<0,005$ gegen akuten Diabetes.

6) $2 p<0,001$ gegen Kontrolle und akuten Diabetes.

7) $2 \mathrm{p}<0,005$ gegen chronischen Diabetes.

8) $2 \mathrm{p}<0,01$ gegen chronischen Diabetes.

9) Nicht signifikant gegen chronischen Diabetes; $2 \mathrm{p}<0,02$ gegen Kontrolle; $2 p<0,01$ gegen chronischen Diabetes mit Teiltherapie.

10) $2 \mathrm{p}<0,001$ gegen chronischen Diabetes; $2 \mathrm{p}<0,05$ gegen Kontrolle; Nicht signifikant gegen chronischen Diabetes mit Teiltherapie.
Die RNA-Synthese, gemessen mit Hilfe des Einbaues von Uridin, ist im akuten Diabetes auf ein Drittel der Kontrollwerte vermindert (Tab. 4). Wie beim Lyin-Einbau findet sich eine signifikante $(2 p<0,005)$ Steigerung durch Insulin in vitro in den Glomerula von Normaltieren, während sich dieser Effekt im akuten Diabetes nicht nachweisen läßt. Chronisch diabetische Tiere weisen einen signifikant $(2 p<0,001)$ erhöhten Uridin-Einbau der isolierten Glomerula auf, der durch die Insulinbehandlung wieder den Kontrollwerten angenähert wird (Tab. 4).

Der Einbau von Lysin und Uridin war in den Glomerula von Normaltieren abhängig von der Glucosekonzentration bis zu $10 \mathrm{mmol} / 1$ (Tab. 5). Dabei waren die Einbauraten bei einer Glucosekonzentration von $10 \mathrm{mmol} / \mathrm{l} \mathrm{je-}$ weils signifikant verschieden von den Einbauraten bei niedrigeren Glucosekonzentrationen.

\section{Diskussion}

Bei Untersuchungen an isolierten Zellsystemen ist neben der Einheitlichkeit des Systems die Integrität und Vitalität der Zellen nach morphologischen und metabolischen Kriterien zu beachten. Bei der hier verwendeten Dissoziation des Gewebes wurde im Gegensatz zu anderen Untersuchern auf die Zentrifugation zur Anreicherung der Glomerula verzichtet, weil sich bereits in den Vorversuchen herausstellte, daß wiederholte Zentrifugation das glomeruläre Zellsystem schädigt. Durch die einfache Sedimentation in der Kälte verringerte sich der Anteil der trypanblaugefärbten Glomerula auf $5 \%$.

Die elektronenmikroskopische Untersuchung bestätigte den guten Erhaltungszustand des Zellsystems. Auch die metabolischen Parameter, z. B. Glucose-Oxidation und Lysin-Einbau, zeigten Linearität in Abhängigkeit von der Zeit und der eingesetzten Gewebemenge.

In Ubereinstimmung mit anderen Untersuchern (23), die eine Vergrößerung und einen vermehrten Kollagengehalt

Tab. 5. Abhängigkeit des Lysin- und Uridineinbaues von der Glucosekonzentration des Inkubationsmediums.

\begin{tabular}{lll}
\hline $\begin{array}{l}\text { Glucose } \\
(\mathrm{mmol} / \mathrm{l})\end{array}$ & $\begin{array}{l}\text { Lysin } \\
(\mathrm{nmol} / 1000 \text { Glomerula } \\
\times \mathrm{h})\end{array}$ & $\begin{array}{l}\text { Uridin } \\
(\mathrm{Bq} / 1000 \text { Glomerula } \\
\times \mathrm{h})\end{array}$ \\
\hline 2 & $\begin{array}{l}0,065 \pm 0,006 \\
(\mathrm{n}=5)\end{array}$ & $\begin{array}{l}5,43 \pm 0,55 \\
(\mathrm{n}=5)\end{array}$ \\
5 & $\begin{array}{l}\left.0,071 \pm 0,004^{1}\right) \\
(\mathrm{n}=5)\end{array}$ & $\begin{array}{l}\left.5,37 \pm 0,28^{1}\right) \\
(\mathrm{n}=5)\end{array}$ \\
10 & $\begin{array}{l}\left.0,086 \pm 0,005^{2}\right) \\
(\mathrm{n}=8)\end{array}$ & $\begin{array}{l}\left.6,30 \pm 0,13^{3}\right) \\
(\mathrm{n}=5)\end{array}$ \\
\hline
\end{tabular}

1) Nicht signifikant gegen $2 \mathrm{mmol} / \mathrm{l}$ Glucose.

2) $2 \mathrm{p}<0,001$ gegen $2 \mathrm{mmol} / 1$ und $5 \mathrm{mmol} / 1$ Glucose.

3) 2 p $<0,01$ gegen $2 \mathrm{mmol} / 1$ Glucose und $2 \mathrm{p}<0,001$ gegen $5 \mathrm{mmol} / 1$ Glucose. 
der Glomerula im Diabetes fanden, ergaben unsere Untersuchungen eine Verdoppelung des DNA-Gehaltes und eine darüber hinausgehende Vermehrung des Proteingehaltes der isolierten Glomerula im chronischen Diabetes, während im akuten Diabetes noch keine Veränderungen des DNA- bzw. Proteingehaltes gegenüber den Kontrollen nachzuweisen waren.

Die auf Befunden an Autopsie-Nieren beruhende These, daß es sich bei der Vergrößerung der Glomerula um eine kompensatorische Hypertrophie zum Ersatz zerstörter Glomerula handeln könnte (24), wird durch die schnelle Normalisierung des DNA- und Proteingehaltes unter der Insulintherapie bei den chronisch diabetischen Tieren in Frage gestellt. Wenn ein Teil der Glomerula wegen einer Verlegung der Kapillarschlingen funktionell nicht mehr aktiv wäre, dann müßte die kompensatorische Hypertrophie auch unter der Insulintherapie zum Erhalt der Nierenfunktion weiter bestehen bleiben.

Der Rückgang des DNA- und Proteingehaltes auch unter der verzögerten Therapie nach therapiefreiem Intervall zeigt, daß eine vermehrte Zellinfiltration als einzige Ursache der erhöhten DNA- und Proteingehalte ausscheidet. Vielmehr sind die genannten Veränderungen insulinabhängig und müssen auf die Hyperglykämie, die Glucosurie, die Proteinurie und/oder den Insulinmangel zurückgeführt werden.

Die erhobenen Befunde veranlassen erneut zu kritischen Überlegungen bezüglich des ,,richtigen“ Bezugssystems. Bezieht man die gefundenen Einbauraten von Lysin und Uridin auf die Zahl der eingesetzten Glomerula, so werden die Befunde von Cohen \& Khalifa (12) über die gesteigerte Proteinsynthese in isolierten Glomerula im Dia- betes bestätigt und lassen sich auch für die RNA-Synthese nachweisen. Berücksichtigt man jedoch die Verdoppelung des DNA-Gehaltes und die Vermehrung des Proteingehaltes auf das Zweieinhalb fache, so ergibt sich eine Verringerung der Einbauraten von Lysin und Uridin bezogen auf diese Parameter (Tab. 6 und 7). Daraus ist zu folgern, daß vermehrt Strukturproteine abgelagert worden sind, was dem morphologischen Korrelat der Basalmembranverdickung entsprechen könnte. Der Bezug der metabolischen Zelleistuing auf das Gesamtprotein oder das Trokkengewicht erscheint daher problematisch.

Auch die Bezugsgröße DNA ist angesichts der beschriebenen Zellvermehrungen in den Glomerula in Frage gestellt. Daher wurden unsere Befunde auf die Zahl der eingesetzten Glomerula bezogen.

Aus früheren Untersuchungen ist bekannt, daß im kollagenähnlichen Basalmembranprotein verhältnismäßig viel Lysin enthalten ist (13-17). Da aber gerade die Basalmembranveränderungen im Mittelpunkt des Interesses stehen, ist die Messung des Lysin-Einbaues in das säurefällbare Protein ein geeigneter Parameter zur Messung der Protein- und speziell der Basalmembransynthese.

Während unsere Untersuchungen in Übereinstimmung mit den Befunden von Wahl et al. (18-19) keinen Insulin-Effekt auf die Glucose-Oxidation in isolierten Glomerula ergaben, zeigen die Ergebnisse der Lysin- und Uridin-Einbauversuche, daß der Stoffwechsel des glomerulären Zellsystems insulinsensitiv ist. Dies gilt sowohl fụir die Insulinzugabe in vitro als auch in vivo.

Insulin in vitro steigert den Einbau von Lysin und Uridin in das säurefällbare Protein in Glomerula von Normal-

Tab. 6. Einbau von Lysin bezogen auf die Zahl der Glomerula, den DNA- und Proteingehalt der Glomerula.

\begin{tabular}{|c|c|c|c|}
\hline & $\begin{array}{l}\text { Lysin } \\
\text { (nmol/1000 Glomerula } \times \text { h) }\end{array}$ & $\begin{array}{l}\text { Lysin } \\
\text { (nmol/10 } \mu \mathrm{g} \text { DNA } \times \mathrm{h})\end{array}$ & $\begin{array}{l}\text { Lysin } \\
\text { (nmol/100 } \mu \mathrm{g} . \text { Protein } \times \mathrm{h})\end{array}$ \\
\hline Kontrolle & $\begin{array}{l}0,086 \pm 0,005 \\
(n=8)\end{array}$ & $\begin{array}{l}0,105 \pm 0,006 \\
(n=8)\end{array}$ & $\begin{array}{l}0,052 \pm 0,003 \\
(n=8)\end{array}$ \\
\hline Akuter Diabetes & $\begin{array}{l}0,086 \pm 0,006 \\
(n=5)\end{array}$ & $\begin{array}{l}0,105 \pm 0,007 \\
(n=5)\end{array}$ & $\begin{array}{l}0,052 \pm 0,003 \\
(n=5)\end{array}$ \\
\hline Chronischer Diabetes & $\begin{array}{l}\left.0,105 \pm 0,007^{1}\right) \\
(n=5)\end{array}$ & $\begin{array}{l}\left.0,066 \pm 0,004^{2}\right) \\
(n=5)\end{array}$ & $\begin{array}{l}\left.0,026 \pm 0,002^{2}\right) \\
(n=5)\end{array}$ \\
\hline $\begin{array}{l}\text { Chronischer Diabetes } \\
\text { mit Teiltherapie }\end{array}$ & $\begin{array}{l}\left.0,147 \pm 0,022^{3}\right) \\
(n=5)\end{array}$ & $\begin{array}{l}\left.0,133 \pm 0,020^{4}\right) \\
(n=5)\end{array}$ & $\begin{array}{l}\left.0,063 \pm 0,009^{5}\right) \\
(n=5)\end{array}$ \\
\hline $\begin{array}{l}\text { Chronischer Diabetes } \\
\text { mit Dauertherapie }\end{array}$ & $\begin{array}{l}\left.0,104 \pm 0,018^{6}\right) \\
(n=5)\end{array}$ & $\begin{array}{l}\left.0,091 \pm 0,016^{7}\right) \\
(n=5)\end{array}$ & $\begin{array}{l}\left.0,044 \pm 0,008^{8}\right) \\
(n=5)\end{array}$ \\
\hline
\end{tabular}

1) $2 \mathrm{p}<0,001$ gegen Kontrolle.

$2 \mathrm{p}<0,005$ gegen akuten Diabetes.

2) $2 \mathrm{p}<0,001$ gegen Kontrolle und akuten Diabetes.

3) $2 \mathrm{p}<0,005$ gegen chronischen Diabetes.

$2 \mathrm{p}<0,001$ gegen Kontrolle.

4) $2 \mathrm{p}<0,001$ gegen chronischen Diabetes. $2 \mathrm{p}<0,005$ gegen Kontrolle.

5) $2 \mathrm{p}<0,001$ gegen chronischen Diabetes. $2 p<0,01$ gegen Kontrolle.
6) Nicht signifikant gegen chronischen Diabetes.

$2 p<0,02$ gegen Kontrolle.

$2 p<0,01$ gegen chronischen Diabetes mit Teiltherapie.

7) $2 \mathrm{p}<0,001$ gegen chronischen Diabetes.

${ }^{\circ} 2 \mathrm{p}<0,05$ gegen Kontrolle.

$2 \mathrm{p}<0,01$ gegen chronischen Diabetes mit Teiltherapie.

8) $2 \mathrm{p}<0,005$ gegen chronischen Diabetes.

$2 \mathrm{p}<0,025$ gegen Kontrolle.

$2 p<0,01$ gegen chronischen Diabetes mit Teiltherapie. 
Tab. 7. Einbau von Uridin, bezogen auf die Zahl, den DNA- und Proteingehalt der Glomerula.

\begin{tabular}{|c|c|c|c|}
\hline & $\begin{array}{l}{\left[\mathrm{U}-{ }^{14} \mathrm{C}\right] \text { Uridin }} \\
(\mathrm{Bq} / 1000 \\
\text { Glomerula } \times \mathrm{h})\end{array}$ & $\begin{array}{l}{\left[\mathrm{U}-{ }^{14} \mathrm{C}\right] \text { Uridin }} \\
(\mathrm{Bq} / 10 \mu \mathrm{g} \\
\mathrm{DNA} \times \mathrm{h})\end{array}$ & $\begin{array}{l}{\left[\mathrm{U}^{-14} \mathrm{C}\right] \text { Uridin }} \\
(\mathrm{Bq} / 100 \mu \mathrm{g} \\
\text { Protein } X \mathrm{~h})\end{array}$ \\
\hline Kontrolle & $\begin{array}{l}6,30 \pm 0,13 \\
(n=5)\end{array}$ & $\begin{array}{l}7,72 \pm 0,17 \\
(n=5)\end{array}$ & $\begin{array}{l}3,82 \pm 0,08 \\
(n=5)\end{array}$ \\
\hline $\begin{array}{l}\text { Akuter } \\
\text { Diabetes }\end{array}$ & $\begin{array}{l}\left.2,17 \pm 0,25^{1}\right) \\
(n=5)\end{array}$ & $\begin{array}{l}\left.2,65 \pm 0,30^{1}\right) \\
(n=5)\end{array}$ & $\begin{array}{l}\left.1,32 \pm 0,15^{1}\right) \\
(n=5)\end{array}$ \\
\hline $\begin{array}{l}\text { Chronischer } \\
\text { Diabetes }\end{array}$ & $\begin{array}{l}\left.8,37 \pm 0,45^{2}\right) \\
(n=5)\end{array}$ & $\begin{array}{l}\left.5,23 \pm 0,28^{2}\right) \\
(n=5)\end{array}$ & $\begin{array}{l}\left.2,08 \pm 0,12^{2}\right) \\
(n=5)\end{array}$ \\
\hline $\begin{array}{l}\text { Chronischer } \\
\text { Diabetes mit } \\
\text { Teiltherapie }\end{array}$ & $\begin{array}{l}\left.6,77 \pm 0,95^{3}\right) \\
(n=5)\end{array}$ & $\begin{array}{l}\left.6,10 \pm 0,85^{4}\right) \\
(n=5)\end{array}$ & $\begin{array}{l}\left.2,88 \pm 0,40^{5}\right) \\
(n=5)\end{array}$ \\
\hline $\begin{array}{l}\text { Chronischer } \\
\text { Diabetes mit } \\
\text { Dauertherapie }\end{array}$ & $\begin{array}{l}\left.6,12 \pm 0,07^{6}\right) \\
(n=5)\end{array}$ & $\begin{array}{l}\left.5,33 \pm 0,07^{7}\right) \\
(n=5)\end{array}$ & $\begin{array}{l}\left.2,62 \pm 0,03^{8}\right) \\
(n=5)\end{array}$ \\
\hline
\end{tabular}

1) $2 \mathrm{p}<0,001$ gegen Kontrolle.

2) $2 \mathrm{p}<0,001$ gegen Kontrolle und akuten Diabetes.

3) $2 \mathrm{p}<0,01$ gegen chronischen Diabetes.

Nicht signifikant gegen Kontrolle.

4) Nicht signifikant gegen chronischen Diabetes. $2 \mathrm{p}<0,005$ gegen Kontrolle.

5) $2 \mathrm{p}<0,005$ gegen chronischen Diabetes. $2 \mathrm{p}<0,001$ gegen Kontrolle.

6) $2 \mathrm{p}<0,001$ gegen chronischen Diabetes. Nicht signifikant gegen chronischen Diabetes mit Teiltherapie. $2 p<0,05$ gegen Kontrolle.

7) Nicht signifikant gegen chronischen Diabetes. Nicht signifikant gegen chronischen Diabetes mit Teiltherapie. $2 p<0,001$ gegen Kontrolle.

8) $2 \mathrm{p}<0,001$ gegen chronischen Diabetes.

Nicht signifikant gegen chronischen Diabetes mit Teiltherapie. $2 p<0,001$ gegen Kontrolle.

tieren. Glomerula von akut diabetischen Tieren zeigen diesen Insulin-Effekt nicht, d.h. es besteht „Insulinresistenz".

Insulin in vivo führt die erhöhten Einbauraten von Lysin und Uridin in den Normalbereich zurück. Die höheren Einbauwerte für Lysin beim chronischen Diabetes mit Teiltherapie, verglichen mit dem chronischen Diabetes ohne Therapie, beruhen möglicherweise auf einer verminderten metabolischen Leistungsfähigkeit zur Proteinsyn- these der glomerulären Zellen im chronischen Diabetes, so daß dort nur ein Teil der eingesetzten Zellen die volle Stoffwechselak tivität hatte.

Als einziger der von uns untersuchten Stoffwechselparameter ist die Uridin-Einbaurate bereits im akuten Diabetes verändert, und zwar auf ein Drittel der Kontrollwerte herabgesetzt. Als Erklärung könnte einmal eine allgemein toxische oder direkt depressive Wirkung des Streptozotocins auf die RNA-Synthese angenommen werden, oder aber es handelt sich um eine Wirkung des akuten Insulinmangels. Denn nach einer Adaptationszeit lassen sich unter chronischem Insulinmangel erhöhte Einbauraten für das Uridin und damit eine erhöhte RNA-Synthese, bezogen auf die Zahl der eingesetzten Glomerula, nachweisen.

Die erhöhte Protein- und RNA-Synthese und die Normalisierung durch Insulin konnte bisher nur am glomerulären Zellsystem nachgewiesen werden (12). An anderen Zellsystemen zeigt Insulin einen proteinanabolen Effekt. Dem Insulinmangel könnte daher bei der Entwicklung der diabetischen Mikroangiopathie eine pathogenetische Bedeutung zukommen.

An den isolierten Glomerula hatte nicht nur Insulin einen Stoffwechseleffekt. Wahl et al. (19) konnten zeigen, daß die Oxidation und der Einbau von Glucose durch die Erhöhung der Glucosekonzentration im Inkubationsmedium gesteigert werden. Unsere Untersuchungen ergaben, daß auch der Lysin- und der Uridin-Einbau durch die Erhöhung der Glucosekonzentration in vitro gesteigert werden können.

Beim Glucose-Einbau in die Basalmembran wird zu klären sein, ob ein aktiver Einbau von Glucose oder eines Metaboliten erfolgt oder ob eine nichtenzymatische Glucosidierung der Basalmembranproteine abläuft. Die erhöhte Glucosidierung von Proteinen im Diabetes ist bekannt (25-26) und könnte auch bei der Entstehung der diabetischen Mikroangiopathie von Bedeutung sein. Neben dem Insulinmangel kommt somit nach unseren Ergebnissen wahrscheinlich auch der Hyperglykämie eine wichtige Rolle in der komplexen Pathogenese der diabetischen Mikroangiopathie der Niere zu.

\section{Literatur}

1. Østerby Hànsen, R., Lundbaek, K., Steen Olsen, T. \& Ørskov, H. (1967), Lab. Invest. 17, 675-692.

2. Cameron, D. P., Amherdt, M., Leunberger, P., Orci, L. \& Stauffacher, W. (1973), in: Early Diabetes (Camerini-Davalos, R. A. \& Cole, H. S. eds.) Academic Press, New York/London, 257-269.

3. Like, A. A., Lavine, R. L., Poffenbarger, L. \& Chick, W. L. (1972), Am. J. Pathol. 66, 193-224.

4. Spiro, R. G. \& Spiro M. J. (1971), Diabetes 20, 641-648.

5. Velasco, C., Oppermann, W., Marine, N. \& Camerini-Davalos, R. A. (1974), Horm. Metab. Res. 6, 427.

6. Khalifa, A. \& Cohen, M. P. (1975), Biochim. Biophys. Acta $386,332-339$.

7. Cohen, M. P. \& Khalifa, A. (1977), Biochim. Biophys. Acta 496, 88-94.

8. Grant, M. E., Harwood, R. \& Williams, I. F. (1976), J. Physiol. 257, $56 \mathrm{P}-57 \mathrm{P}$

9. Risteli, R., Koivisto, V. A., Akerblom, H. K. \& Kivirikko, K. I. (1976), Diabetes 25, 1066-1070.

10. Peterson, D. T., Greene, W. C. \& Reaven G. M. (1971), Diabetes $20,649-654$.

11. Cohen, M. P. \& Vogt, C. A. (1975), Horm. Metab. Res. 7, $338-342$.

12. Cohen, M. P. \& Khalifa, A. (1978), Biochim. Biophys. Acta $500,395-404$.

13. Beisswenger, P. J. \& Spiro, R. G. (1973), Diabetes $22,180-$ 193.

14. Westberg, N. G. \& Michael, A. F. (1973), Acta Med. Scand. 194, 39-47.

15. Kefalides, N. A. (1974), J. Clin. Invest. 53, 403-407. 
16. Sato, T., Munakata, H., Yoshinaga, K. \& Yosizawa, Z. (1975), Clin. Chim. Acta 61, 145-150.

17. Beisswenger, P. J. (1976), J. Clin. Invest. 58, 844-852.

18. Wahl, P., Krezdorn, W. \& Deppermann, D. (1970), Verh. Dtsch. Ges. Inn. Med. 76, 393-396.

19. Wahl, P., Deppermann, D., Deschner, W., Fuchs, E. \& Rexroth, W. (1973), in: Early Diabetes (Camerini-Davalos, R. A. $\&$ Cole, H. S. eds.) Academic Press, New York/London, 147153.

20. Krakower, C. A. \& Greenspon, S. A. (1951), Arch. Pathol. $51,629-639$.
21. Lowry, O. H., Rosebrough, N. J., Farri, A. L. \& Randall, R J. (1951), J. Biol. Chem. 193, 265-275

22. Burton, K. (1956), Biochem. J. 62, 315-323.

23. Klein, L., Butcher, D. L., Sudilovsky, O., Kikkawa, R. \& Miller, M. (1975), Diabetes 24, 1057-1065.

24. Gundersen, H. J. G. \& Osterby, R. (1977), Diabetologia 13, 43-48.

25. Kennedy, A. L., Kandell, T. W. \& Merimée, T. J. (1979), Diabetes 28, 1006-1010.

26. McFarland, K. F., Catalano, E. W., Day, J. F., Thorpe, S. R. \& Baynes, J. W. (1979), Diabetes 28, 1011-1014.

Prof. Dr. med. H. Reinauer Lehrstuhl für klin. Biochemie der Univer sität Düsseldorf Auf'm Hennekamp 65 4000 Düsseldorf 1 